\title{
CORRELAÇÃO ENTRE O USO DE ESTATINAS E A PREVENÇÃ̃O E PROGNÓSTICO DE PACIENTES ACOMETIDOS POR SEPSE
}

\author{
CORRELATION BETWEEN THE USE OF STATINS AND THE \\ PREVENTION AND PROGNOSIS OF SEPSIS PATIENTS
}

Hélida Maravilha Dantas e Sousa Almeida ${ }^{1}$ Ana Cecília Alexandre dos Ramos ${ }^{2}$ Sávio Benvindo Ferreira ${ }^{3}$

RESUMO: OBJETIVO: Analisar a participação das estatinas na sepse. MÉTODO: Consiste em um estudo descritivo-exploratório, com abordagem qualitativa, adotando como metodologia a revisão integrativa da literatura. A pesquisa foi realizada na Biblioteca Virtual em Saúde, utilizando os descritores estatinas; sepse. A obra foi estruturada com 11 artigos, dos últimos 5 anos, que respondiam aos critérios propostos, nos idiomas inglês, português e espanhol. RESULTADOS: A sepse é uma problemática mundial e atual, consistindo em uma resposta inadequada do organismo a uma infecção. Este problema provoca grande morbidade devido o comprometimento extenso a órgãos vitais, como coração e cérebro, resultando comumente em óbitos ou sequelas. Novas alternativas vêm sendo estudadas para a terapêutica preventiva e até mesmo protetora contra a sepse. As estatinas são uma classe farmacológica hipolipemiante, que possui cada vez mais destaque por seus efeitos pleitropicos, como a capacidade imunomoduladora e neuroprotetora. Os estudos atuais demonstram que essa classe de fármaco possui promissoras propriedades úteis no manejo da sepse, desde a proteção da barreira hematoencefálica, diminuição do comprometimento sistêmico e aumento da sobrevida e menor tempo de internação. CONCLUSÃO: As estatinas podem ser discutidas e consideradas na terapêutica contra a sepse, desde a prevenção de danos orgânicos, até a diminuição de problemáticas após a instalação do problema. Mesmo existindo a necessidade de mais estudos, é uma alternativa que já pode ser considerada na prática clínica após avaliação individual dos pacientes.

\footnotetext{
${ }^{1}$ Graduanda em Enfermagem da Universidade Federal de Campina Grande, do Centro de Formação de Professores.

${ }^{2}$ Graduanda em Enfermagem da Universidade Federal de Campina Grande, do Centro de Formação de Professores.

${ }^{3}$ Professor Doutor da Unidade Acadêmica de Ciências da Vida da Universidade Federal de Campina Grande, do Centro de Formação de Professores.
} 
Palavras chave: Inibidores de Hidroximetilglutaril-CoA Redutases; Sepse; Terapêutica.

\begin{abstract}
OBJECTIVE: To analyze the participation of statins in sepsis. METHOD: This is a descriptive and exploratory study with a qualitative approach, adopting the integrative literature review as a methodology. The research was performed at the Virtual Health Library, using the statin descriptors; sepsis The work was structured with 11 articles from the last 5 years, which met the proposed criteria, in English, Portuguese and Spanish. RESULTS: Sepsis is a worldwide and current problem, consisting of an inadequate response of the organism to an infection. This problem causes high morbidity due to extensive impairment to vital organs such as the heart and brain, commonly resulting in death or sequelae. New alternatives are being studied for preventive and even protective therapy against sepsis. Statins are a hypolipemic drug class that is increasingly highlighted for its pleitropic effects, such as immunomodulatory and neuroprotective capacity. Current studies show that this class of drug has promising useful properties in sepsis management, from protection of the blood-brain barrier, decreased systemic impairment and increased survival and shorter hospital stay. CONCLUSION: Statins can be discussed and considered in sepsis therapy, from the prevention of organ damage to the reduction of problems after the onset of the problem. Even if there is a need for further studies, it is an alternative that can already be considered in clinical practice after individual assessment of patients.
\end{abstract}

Keywords: Hydroxymethylglutaryl-CoA Reductase Inhibitors; Sepsis; Therapeutics. 


\section{INTRODUÇÃO}

As doenças crônicas não transmissíveis ganham cada vez mais espaço na sociedade, devido a vários fatores associados a vida moderna e a genética, a exemplo são as patologias que atinge o sistema cardiovascular. A aterosclerose, uma doença característica da fase adulta e idosa, descrita como uma inflamação crônica na camada mais interna das artérias, provocando uma estenose. Geralmente esse processo ocorre devido ao acúmulo de lipoproteínas na parede dessas artérias, gerando o que é conhecido como placa de ateroma ou aterosclerótica. A estrutura geral de uma lipoproteína possui uma porção composta por uma apoproteína, e colesterol, triglicerídeos e fosfoglicerídeos formando a parte lipídica. Essas moléculas auxiliam no transporte de gordura pelo plasma, e possuem algumas classificações segundo suas propriedades, como características biofísicas e estruturais (FORTI, DIAMENT 2006; MARANHÃO et al., 2014).

Com base nessa categorização, podem ser pontuadas como exemplo o LDL, VLDL e HDL. As lipoproteínas de baixa densidade (LDL), transportam o colesterol para diversas partes do corpo, para desempenharem diversas funções orgânicas como a produção de esteroides. O VLDL corresponde as lipoproteínas de muito baixa densidade, que são sintetizados no fígado e transportadores de triglicerídeos pela corrente sanguínea. As lipoproteínas de alta densidade (HDL), responsáveis por levar o colesterol ao fígado, sendo associado a proteção contra a formação de placas de ateroma. A elevação do colesterol e triacilglicerol plasmático, assim como níveis altos de LDL e VLDL também contribuem na gênese desse problema. Essa condição provoca grandes problemáticas ao organismo humano, de forma sistêmica, como a hipertensão arterial sistêmica (HAS), doença cardíaca coronária, infarto agudo do miocárdio (IAM), acidente vascular encefálico (AVE) (JÚNIOR, 2016).

O aumento nos níveis séricos de colesterol, LDL, triglicérides, e a diminuição do HDL, consiste em uma condição denominada dislipidemia. Esse problema pode ter causas genéticas, relacionadas a hábitos alimentares e estilo de vida pouco 
saudável, como o elevado consumo de gorduras e a inatividade física. Como essa condição está intimamente associada com a aterosclerose, e outros problemas cardiovasculares, a sua prevenção com a adoção de uma nutrição adequada e atividade física é bastante disseminada. Além disso, o número de casos de dislipidemias é crescente em todo o mundo, sendo ainda associado a terapêutica do problema alguns medicamentos hipolipemiantes, como as estatinas (DAVIDSON, 2019; GUPTA, MALHOTRA, 2014).

Esses fármacos agem inibindo seletivamente e reversivelmente a redutase 3hidroxi-3-metilglutaril-coenzima A (HMG-CoA) e, pesquisas recentes, vêm revelando o potencial dessas drogas sob outros aspectos. Exemplo disso é a participação em processos inflamatórios, desempenhando atividade supressora, como o decréscimo da produção da proteína C reativa, e de citocinas pro-inflamatória. Outros estudos apresentam ainda descobertas consideráveis sobre os efeitos das estatinas na sepse - que são manifestações graves a uma infecção, podendo levar até a falência múltipla dos órgãos (VIASUS et al., 2015; GRUDZINSKA et al., 2017).

Essa doença agrava substancialmente a condição de saúde do enfermo, levando a morbidade, maiores períodos de internação hospitalar, chegando a gerar consequências ao organismo, sendo uma condição que está aumentando a cada ano. Assim, o descobrimento de aplicações inovadoras para essa classe de fármacos desempenha um potencial preventivo e protetor contra esse problema, sendo necessários, no entanto, maiores investigações sobre os efeitos das estatinas na sepse são necessárias para subsidiar discussões sobre a aplicação clínica dessas drogas (MARTIN et al., 2003; GUI, YANG, ZHANG, 2017).

Nesse contexto, a presente pesquisa objetiva analisar a participação das estatinas na sepse. A investigação se faz necessária para discutir inovações clínicas no manejo dessa problemática, a partir de uma busca bibliográfica pela literatura científica atual, concedendo a profissionais da saúde uma visão mais crítica acerca desses aspectos, declamando essa problemática sob uma outra ótica, e abrindo espaço para novas alternativas investigativas e clínicas. 


\section{METODOLOGIA}

Este trabalho consiste em um estudo descritivo-exploratório, com abordagem qualitativa, utilizando o artifício metodológico de revisão integrativa que emprega a pesquisa bibliográfica como processo de documentação indireta. Para a realização do estudo, consideraram-se as seguintes etapas necessárias para sua execução:

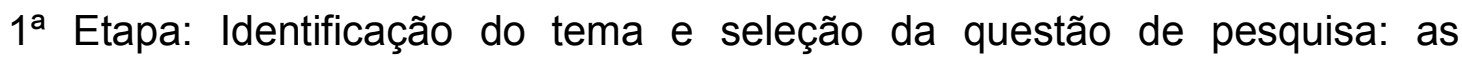
infecções são problemáticas que provocam grandes discursões mundiais e atemporais. A sepse está associada a uma significativa morbimortalidade, e as estratégias que vislumbram entender melhor esse quadro e fornecer meios que minimizem os danos vêm sendo cada vez mais explorados. Os estudos vêm demonstrando a participação das estatinas no sistema imunológico e na terapêutica de infecções, especialmente no que tange a sepse. Nesse sentido, para guiar a pesquisa, formulou-se a seguinte questão norteadora: "O que a literatura aponta sobre a participação das estatinas em sepse?".

$2^{\mathrm{a}}$ Etapa: Adoção dos critérios de inclusão e exclusão: o levantamento bibliográfico foi realizado nos meses de dezembro de 2019, por meio de uma busca de publicações indexadas nas bases de dados: Scientific Electronic Library (SciELO), Sistema Online de Busca e Análise de Literatura Médica (MEDLINE), Literatura Latino-Americana e do Caribe em Ciências da Saúde (LILACS) através Biblioteca Virtual em Saúde (BVS) pelo cruzamento dos descritores: Estatinas; Sepse; mediante o uso do operador booleano "AND".

Os critérios de inclusão foram: pesquisas que abordassem o envolvimento desses fármacos em processos de sepse, com ênfase na prevenção de danos causados por essa problemática. Apresentar publicação como artigos originais, na íntegra, indexados nas bases de dados propostas, disponíveis em português, inglês e espanhol, nos últimos 5 anos, cujos textos retratassem a temática. Quanto aos critérios de exclusão: trabalhos que não se apresentassem na íntegra nas bases de dados e nas bibliotecas pesquisadas, como também aqueles que não coincidiam 
com a temática proposta, livros, revisões bibliográficas, editoriais, dissertações e tese.

$3^{a}$ Etapa: Identificação dos estudos pré-selecionados e selecionados: após o cruzamento dos descritores obteve-se um somatório de 185 artigos. Com o primeiro refinamento da pesquisa foram identificadas 59 obras, sendo pré-selecionadas 14 estudos pela leitura dos títulos e resumos. Após a leitura dos artigos na íntegra, apenas 11 foram selecionados, dos anos de 2014, 2015, 2016, 2017 e 2019, por serem condizentes com a questão norteadora do presente estudo.

$4^{a}$ Etapa: Categorização dos estudos selecionados: foram analisados os resultados de cada artigo individualmente e, em seguida, realizou-se uma análise temática comparativa sobre os resultados encontrados. Para subsidiar as discussões e interpretação dos resultados obtidos a partir dos artigos selecionados na revisão, foi executado uma análise de referências dos estudos encontrados nas bases de dados selecionadas.

$5^{a}$ Etapa: Análise e interpretação dos resultados: de maneira minuciosa, observando os dados encontrados em cada estudo e as conclusões que cada autor apresentou, foi verificado as possíveis contribuições para elucidar a questão norteadora da presente pesquisa.

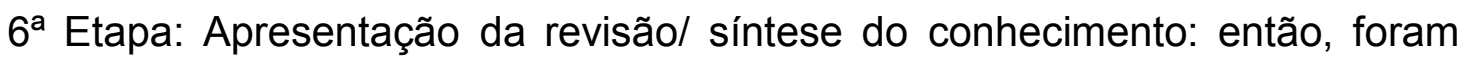
levantadas as conclusões a partir do que foi evidenciado em cada estudo, realizando uma reflexão a respeito das respostas obtidas para atingir o objetivo proposto, produzindo uma interrelação entre as obras.

\section{RESULTADOS E DISCUSSÕES}

A sepse é uma problemática mundial e cada vez mais cresce as investigações sobre sua gênese assim como formas de prevenção e proteção contra agravos. Antigamente era conhecida como infecção generalizada, e sua manifestação é decorrida de uma infecção localizada em qualquer órgão e que o organismo responde inadequadamente, levando a um mau funcionamento 
fisiológico, devendo ser descoberta e tratada rapidamente para evitar a morte, já que esta é uma das principais causas de óbito por infecção (KIM; PARK, 2019).

Como forma de combate à essas manifestações, estudos empregando estatinas apresentaram uma diminuição ao risco de adquirir sepse em diferentes quadros clínicos. Estes resultados abriram espaço para serem intensificados os trabalhos que avaliassem melhor esse funcionamento. A obra de Yeh et al. (2019) avaliaram a associação entre o uso dessas drogas com o risco de sepse em indivíduos com demência, já que pesquisas apontaram certa participação protetora desses fármacos em patologias neurodegenerativas (como o Parkinson e Alzheimer). Para isso, foi feito uma pesquisa retrospectiva na National Health Insurance Research Database, em Taiwan. Foram identificados 308 pacientes com idade acima de 40 anos de idade e recém-diagnosticados com demência, que usaram estatina após esse diagnóstico. A análise não revelou resultados positivos quanto o uso de estatinas em pacientes com demência e o risco de sepse.

Outra pesquisa, realizada com 36 ratos pré-tratados com sinvastatina com o escopo de investigar o efeito protetor da sinvastatina na depressão miocárdica devido a sepse, identificaram uma diminuição significativa nos níveis de citocinas pró-inflamatórias, como TNF- $\alpha$, IL-1 $\beta$, IL-6 nos tecidos do miocárdio (WANG et al., 2017). Tais resultados são animadores, visto que muitos pacientes com sepse falecem devido a depressão miocárdica causando uma insuficiência cardíaca, apresentando uma alternativa preventiva em casos de sepse, podendo diminuir a morbidade e a mortalidade nesses casos.

Ainda no que compete os efeitos protetores contra a sepse, os pesquisadores Gui, Yang e Zhang (2017), avaliaram na perspectiva da população idosa hospitalizada com infecções bacterianas. Para tanto, foi desenvolvido um estudo de coorte retrospectivo, com 257 idosos chineses hospitalizados, divididos em grupos para a investigação. O número de disfunções orgânicas nos indivíduos tratados com estatinas foi de $13,3 \%$, versos $31,2 \%$ dos não tratados com a droga, um valor significativamente menor, indicando que ocorreu uma redução no desenvolvimento de sepse e disfunções relacionadas a infecção. Essa pesquisa não mostrou diminuição da mortalidade e da incidência de internações em unidades de terapia intensiva ou tempo de internação. Apesar de não representar uma significativa 
alternativa, segundo esta obra, no que tange a mortalidade, a diminuição distúrbios orgânicos e sepse garantem maior bem-estar aos enfermos, além de poder ser considerado uma mudança de prognóstico.

A avaliação de aspectos bioquímicos envolvidos na sepse foi explorada por Morel e colaboradores (2017), a partir do estudo mitocondrial em um modelo de sepse em ratos. Esses animais receberam um tratamento por sonda de sinvastatina, sendo administradas antes ou depois de instalar o modelo infeccioso (indução de peritonite fecal). O grupo controle foi realizado com animais sépticos com administração de veículo. A terapia com sinvastatina impediu a queda no consumo mitocondrial de oxigênio nas fibras musculares, em ambos tratamentos, sendo paralelo a recuperação de genes relacionados ao metabolismo dos ácidos graxos. Apesar desses resultados terem sido apresentados nos dois grupos, $43,7 \%$ dos ratos pré-tratados com o fármaco sobreviveram setenta e duas horas, em comparação com apenas $12,5 \%$ tratados com estatinas após a instalação da patologia. Sendo assim, a adoção da terapêutica anterior a instalação da infecção pode apresentar maior expectativa de vida aos acometidos pelo problema.

Ainda sobre estudos realizados em modelos pré-clínicos, foi investigado a disfunção cerebral e como prevenir esses danos, visto que acarreta consequências cognitivas aos indivíduos que obtiveram sepse, contribuindo para a morbimortalidade. Uma investigação induzindo sepse em camundongos e aplicando estatinas por via oral, visando a avaliar a prevenção do comprometimento cognitivo após a sepse, a neuro inflamação e disfunções microcirculatória. $O$ uso de atorvastatina/sinvastatina impediu disfunções cognitivas para memórias contextuais hipocampais e dependentes da amídala. A análise bioquímica revelou menores níveis de citocinas e quimiocinas pró-inflamatórias sistêmicas e no tecido cerebral, assim como uma menor ativação microglial. A peroxidação lipídica cerebral e os níveis de mieloperoxidase foram diminuídos, o que representa um aspecto positivo, visto que esse processo e essa enzima são responsáveis pela produção de radicais livres, que em excesso causam danos teciduais. O comprometimento do fluxo sanguíneo foi revertido pelo tratamento com estatinas, assim como a restauração da resposta vasodilatadora colinérgica. Esse estudo não apenas revelou que há uma capacidade neuroprotetora das estatinas em sepse, impedindo o declínio cognitivo e 
reduzindo a inflamação neuronal, como também mantendo a microvascularização adequada no cérebro (REIS et al., 2017).

Foram avaliadas também as propriedades dos HMG-CoA redutase foram avaliadas em pacientes da unidade de terapia intensiva cirúrgica (UTI), com exposição prévia a esses fármacos e avaliado o desenvolvimento de sepse. $O$ percentual de pacientes que desenvolveram sepse mesmo com o uso de estatina foi de $9,77 \%$ contra $33,6 \%$ dos que não utilizaram o fármaco (SCHURR et al., 2016). Os estudos de Beed et al. (2016) também avaliaram o funcionamento do uso prévio das estatinas em pacientes de UTI, no contexto da sobrevida em casos de sepse e pósoperatórios, os pacientes para quais eram prescritos essas drogas antes da admissão na unidade eram mais velhos e tinham mais comorbidades pré-existentes. Nesse estudo a diferença não foi considerada significante, sendo de $9 \%$ contra 15\% do grupo que não utilizou o fármaco, sendo assim não foi associado pelos autores a melhores resultados. Entretanto, quando confrontado os resultados dessas duas obras sob a mesma perspectiva observamos que, esses fármacos podem apresentar resultados promissores quando associados ao tratamento prévio, devendo considerar questões como a maior idade e o maior número de comorbidades do último estudo mencionado para uma análise mais crítica dos dados.

Wang et al. (2016), objetivaram determinar a associação entre o uso crônico de estatinas e sepse a longo prazo, utilizados dados de participantes adultos, com idade igual ou superior a 45 anos, acompanhados por 10 anos (2003 a 2012). Esse estudo se diferencia dos demais por analisar um grande grupo populacional em comunidade por um longo intervalo temporal, não limitando a um cenário de internações hospitalares nem comorbidades significativas. Foi detectada uma não associação do uso do medicamento à presença de sepse grave ou mortalidade hospitalar por sepse, sendo resultados promissores quando discutidos formas de prevenção primária à patologia.

O tempo de internação e readmissões em pacientes com sepse hospitalizados foi avaliado à luz da associação dos HMG-CoA redutases, a partir de um estudo retrospectivo com adultos diagnosticados com sepse. Os pacientes que fizeram uso de estatinas durante a hospitalização foram associados ao menor tempo de permanência na internação, com uma diferença média de 1,55 dias, apesar de 
não afetar a frequência de readmissões (NKEMDIRIM OKERE, RENIER; 2015). O menor tempo de internação é crucial para garantir um melhor bem- estar ao paciente, além de ser um fator que previne maiores agravantes, já que o ambiente hospitalar é geralmente desconfortante e com vários fatores que favorecem o surgimento de comorbidades, como o risco de infecções hospitalares. Esses dados revelam um inovador propósito a ser considerado na terapêutica.

Como a sinvastatina possui uma potente capacidade anti-inflamatória e antioxidante, e a inflamação e o estresse oxidativo são fatores que influenciam diretamente a perda da integridade da barreira hematoencefálica (BHE) e que estão presentes na sepse, o uso desse fármaco poderia atuar amenizando esse dano. A partir dessa premissa, os pesquisadores Yang et al. (2015) buscaram avaliar em seus estudos se tal capacidade era visualizada utilizando como percurso metodológico um modelo de sepse em ratos. Foi observado uma que a droga foi capaz de atenuar os danos a BHE, como a diminuição da sua permeabilidade e do edema cerebral, comparado aos animais que não consumiram o medicamento. Assim, é melhor explicado o possível mecanismo neuroprotetor das estatinas, como mencionado por Reis et al. (2017), que em sua obra, revelaram seu impedimento no declínio cognitivo, podendo correlacionar esses estudos, visando diminuir os danos neurológicos conseguintes da sepse.

As estatinas possuem uma classificação, atribuída pelo American College of Cardiology e o American Heart Association, como inibidores HMG-CoA de baixa e alta potência que podem ter respostas diferentes quando aplicados no manejo da sepse. Um estudo foi efetivado com pacientes hospitalizados em Taiwan para realizar essa averiguação, sendo divididos em usuários de estatina de alta potência (com pelo menos $10 \mathrm{mg}$ de rosuvastatina, pelo menos $20 \mathrm{mg}$ de atorvastatina ou pelo menos $40 \mathrm{mg}$ de sinvastatina), usuários de baixa potência (todos os outros tratamentos com estatina) e não usuários. Os resultados foram promissores, pois detectou que o uso prévio de estatina por pelo menos 30 dias estava levou a redução de $14 \%$ na morte hospitalar. Além de apreciar os efeitos protetores das drogas em questão, que indicou certa persistência 1 ano após a alta. Quanto a distinção dos resultados relacionados a classificação, as estatinas de alta potência 
reduziram o risco em $20 \%$ de mortalidade em 1 ano após a sepse, e as de baixa potência foram associadas a um risco reduzido de 11\% (OU et al., 2014).

\section{CONSIDERAÇÕES FINAIS}

A sepse é uma problemática mundial e atual, que cresce a cada ano, sendo o motivo de grande morbidade, levando a falência de vários órgãos e podendo resultar em óbito. O uso de estatinas como alternativa preventiva e protetora contra os efeitos da sepse, obtiveram resultados favoráveis e relevantes em boa parte dos estudos realizados até o momento. Pode ser destacado o efeito neuroprotetor e o aumento da sobrevida dos acometidos pelo problema. Essa ênfase pode ser considerada já que em sepse, o prejuízo do sistema nervoso é evidenciado e eleva a morbidade do paciente, o tornando cada vez mais debilitado e aumentando as chances de óbito. E o aumento da sobrevida com o menor número de sequelas possíveis, interfere também na economia, dado o menor período de internação necessário e de insumos para reabilitações.

Foi observado que, a supressão de vias de síntese de proteínas próinflamatórias provocadas pelos inibidores HMG-CoA redutases são meios protetores de vários sistemas durante modelos de sepse, esse resultado fornece melhor embasamento científico para associações futuras. Mesmo assim, o uso dessa farmacoterapia pode ser discutido clinicamente no manejo da sepse, com a necessidade de maior capacitação profissional e discussão das condições do paciente, levantando os fatores pró e contras e avaliando cada caso individualmente, assim, é esperado uma melhor recuperação de indivíduos gravemente debilitados. Entretanto, é evidenciado, na maioria das obras investigadas, a necessidade de mais estudos que elucidem essa relação, em virtude de melhor esclarecimento clínico e metabólico, como explicar melhor os mecanismos protetores farmacológicos. 


\section{REFERÊNCIAS BIBLIOGRÁFICAS}

BEED, Martin et al. The association between prior statin use and long-term outcomes after critical care admission. Jour. of critic. Ca., v. 35, p. 63-68, 2016. Disponível em: https://linkinghub.elsevier.com/retrieve/pii/S0883-9441(16)30057-0. Acesso em: 04 Jan. 2020.

FORTI, Neusa; DIAMENT, Jayme. Lipoproteínas de alta densidade: aspectos metabólicos, clínicos, epidemiológicos e de intervenção terapêutica. Atualização para os clínicos. Arq. Bras. Cardiol., São Paulo, v. 87, n. 5, p. 671-679, Nov. 2006. Disponível em: $<$ http://www.scielo.br/scielo.php?script=sci_arttext\&pid=S0066-

782X2006001800019\&Ing=en\&nrm=iso>. Acesso em: 07 Jan. 2020.

DAVIDSON, Michael H. Dyslipidemia: (Hyperlipidemia). In: Merck Manual for the Profess. [S. I.], 2019. Disponível em: www.merckmanuals.com / lipid disorders / dyslipidemia .html. Acesso em: 4 jan. 2020.

GRUDZINSKA, Frances S. et al. Statin therapy in patients with community-acquired pneumonia. Clin. Medic., v. 17, n. 5, p. 403-407, 2017. Disponível em: https://www.ncbi.nlm.nih.gov/pmc/articles/PMC6301923/. Acesso em: 04 Jan. 2020.

GUI, Qifeng; YANG, Yunmei; ZHANG, Jiajia. Effects of statins on the development of sepsis and organ dysfunction in hospitalized older patients in China. Braz J Infect Dis, Salvador, v. 21, n. 3 , p. 255-262, June 2017. Disponível em: $<$ http://www.scielo.br/scielo.php?script=sci_arttext\&pid=S141386702017000300255\&lng=en\&nrm=iso>. Acesso em: 04 Jan. 2020.

GUPTA, Puneeta; MALHOTRA, Pavan. New Horizons in Management of Dyslipidemia. JK Scien., $\quad$ v. $16, \quad$ n. 4 , p. $188,2014 . \quad$ Disponível em: https://www.jkscience.org/archives/Dr\%20Puneeta.pdf. Acesso em: 04 Jan. 2020.

JÚNIOR, José Walison Mainart. Tratamento da dislipidemia e prevenção da aterosclerose no âmbito da Equipe de Saúde da Família Belvedere, em Montes Claros, Minas Gerais. 2016. Disponível em:

https://www.nescon.medicina.ufmg.br/biblioteca/pesquisa/simples/Tratamento\%20da\%20disl ipidemia $\% 20$ e $\% 20$ preven $\%$ C3\%A7\%C3\%A30\%20da\%20aterosclerose $\% 20$ no $\% 20 \%$ C3\%A2 mbito\%20da\%20Equipe\%20de\%20Sa\%C3\%BAde\%20da\%20Fam\%C3\%ADlia\%20Belveder e,\%20em\%20Montes\%20Claros,\%20Minas\%20Gerais/1030. Acesso em: 04 Jan. 2020.

KIM, Hwan II; PARK, Sunghoon. Sepsis: Early recognition and optimized treatment. Tuberc. and respirat. disea., v. 82, $\mathrm{n} 1, \quad$ p. 6-14, 2019. Disponível em: <https://www.ncbi.nlm.nih.gov/pmc/articles/PMC6304323/>. Acesso em: 04 Jan. 2020.

MARANHAO, Raul Cavalcante et al . Lipoproteína (a): Estrutura, Metabolismo, Fisiopatologia e Implicações Clínicas. Arq. Bras. Cardiol., São Paulo, v. 103, n. 1, p. 76-84, July 2014. Disponível em: <http://www.scielo.br/scielo.php?script=sci_arttext\&pid=S0066782X2014001900011\&lng=en\&nrm=iso>. Acesso em: 07 Jan. 2020.

MARTIN, Greg S. et al. The epidemiology of sepsis in the United States from 1979 through 2000. New Englan. Jour. of Med., v. 348, n. 16, p. 1546-1554, 2003. Disponível em: $<$ https://www.nejm.org/doi/10.1056/NEJMoa022139?url_ver=Z39.88-

2003\&rfr_id=ori\%3Arid\%3Acrossref.org\&rfr_dat=cr_pub\%3Dwww.ncbi.nlm.nih.gov.>Acesso em: 04 Jan. 2020.

MOREL, Jerome et al. Simvastatin pre-treatment improves survival and mitochondrial function in a 3-day fluid-resuscitated rat model of sepsis. Clin. Scien., v. 131, n. 8, p. 747-758, 2017. 
Disponível em: https://portlandpress.com/clinsci/article-lookup/doi/10.1042/CS20160802. Acesso em: 04 Jan. 2020.

NKEMDIRIM OKERE, Arinze; RENIER, Colleen M. Effects of statins on hospital length of stay and all-cause readmissions among hospitalized patients with a primary diagnosis of sepsis. Annal. of Pharmacothera., v. 49, n. 12, p. 1273-1283, 2015. Disponível em: http://journals.sagepub.com/doi/full/10.1177/1060028015603072?url_ver=Z39.88-

2003\&rfr_id=ori:rid:crossref.org\&rfr_dat=cr_pub\%3dpubmed. Acesso em: 04 Jan. 2020.

OU, Shu-Yu et al. Effect of the use of low and high potency statins and sepsis outcomes. Intensi. care med., v. 40, n. 10, p. 1509-1517, 2014. Disponível em: https://www.ncbi.nlm.nih.gov/pubmed/25091791. Acesso em: 04 Jan. 2020.

REIS, Patricia A. et al. Statins prevent cognitive impairment after sepsis by reverting neuroinflammation, and microcirculatory/endothelial dysfunction. Brain, behav., and immu., v. 60 , p. 293-303, 2017. Disponível em: https://linkinghub.elsevier.com/retrieve/pii/S08891591(16)30496-2. Acesso em: 04 Jan. 2020.

SCHURR, James W. et al. Incidence of sepsis and mortality with prior exposure of HMG-COA reductase inhibitors in a surgical intensive care population. Shock: Injury, Inflam., and Sepsis: Laborat. and Clini. Approach., v. 45, n. 1, p. 10-15, 2016. Disponível em: https://insights.ovid.com/article/00024382-201601000-00003. Acesso em: 04 Jan. 2020.

VIASUS, Diego et al. The effect of simvastatin on inflammatory cytokines in communityacquired pneumonia: a randomised, double-blind, placebo-controlled trial. BMJ open, v. 5, n. 1, p. e006251, 2015. Disponível em: https://bmjopen.bmj.com/content/5/1/e006251.long. Acesso em: 04 Jan. 2020.

WANG, Henry E. et al. Chronic statin use and long-term rates of sepsis: a population-based cohort study. Journ. of inten. care med., v. 31, n. 6, p. 386-396, 2016. Disponível em: https://www.ncbi.nlm.nih.gov/pmc/articles/PMC5038131/. Acesso em: 04 Jan. 2020.

WANG, Yu et al. An experimental study of the protective effect of simvastatin on sepsisinduced myocardial depression in rats. Biomed. \& Pharmacot., v. 94, p. 705-711, 2017. Disponivel em: https://linkinghub.elsevier.com/retrieve/pii/S0753-3322(17)32789-0. Acesso em: 04 Jan. 2020.

YANG, Chen-Hsien et al. Simvastatin attenuates sepsis-induced blood-brain barrier integrity loss. Jour. of surg. resear., v. 194, n. 2, p. 591-598, 2015. Disponível em: https://linkinghub.elsevier.com/retrieve/pii/S0022-4804(14)01065-8. Acesso em: 04 Jan. 2020.

YEH, Liang-Tsai et al. Association between Statin Use and Sepsis Risk in Patients with Dementia: A Retrospective Cohort Study. Internat. Journ. of environm. Resear. and public health, v. 16, n. 9, p. 1626, 2019. Disponível em: https://www.mdpi.com/1660-4601/16/9/1626. Acesso em: 04 Jan. 2020. 